TRANSACTIONS OF THE

AMERICAN MATHEMATICAL SOCIETY

Volume 360, Number 12, December 2008, Pages 6303-6318

S 0002-9947(08)04486-3

Article electronically published on July 21, 2008

\title{
DETECTING FREE SPLITTINGS IN RELATIVELY HYPERBOLIC GROUPS
}

\author{
FRANÇOIS DAHMANI AND DANIEL GROVES
}

\begin{abstract}
We describe an algorithm which determines whether or not a group which is hyperbolic relative to abelian groups admits a nontrivial splitting over a finite group.
\end{abstract}

\section{INTRODUCTION}

In this paper we are concerned with the problem of determining whether or not a group given by some finite presentation admits a nontrivial free product decomposition (or a nontrivial splitting over a finite subgroup). Of course this problem has no solutions for arbitrary finite group presentations. For example, the group $(\mathbb{Z} / 2 \mathbb{Z}) * G$ is freely indecomposable if and only if $G \cong 1$, but it is impossible to decide whether or not a finitely presented group $G$ is trivial. However, in the presence of some geometry, a positive solution is sometimes possible. One of the most important results in this direction is due to V. Gerasimov [13. In the late 1990s, he proved:

Theorem 1.1 (V. Gerasimov). There is an algorithm that, given a finite presentation of a hyperbolic group, computes the number of ends of the group.

In this paper, we consider relatively hyperbolic groups. (See Section 2 for a definition.) Following Gerasimov's ideas, our main result is:

Theorem 1.2. There exists an algorithm which takes as input a finite presentation of a group $\Gamma$ which is hyperbolic relative to abelian subgroups, and outputs 'Yes' or 'No', according to whether or not $\Gamma$ admits a nontrivial splitting over a finite group.

According to Dunwoody's accessibility, for a given finitely presented group $G$ there is a bound on the size of a graph of groups with finite edge groups and fundamental group $G$. Thus, there is a Dunwoody decomposition of such a $G$, which is the most refined splitting of $G$ as a graph of groups with finite edge groups. Using Theorem 1.2, it is straightforward to deduce the following:

Theorem 1.3. There is an algorithm which takes as input a finite presentation of a group $\Gamma$ which is hyperbolic relative to abelian subgroups, and outputs a presentation for a graph of groups encoding the Dunwoody decomposition of $\Gamma$, together with an isomorphism to $\Gamma$.

Received by the editors October 31, 2006.

2000 Mathematics Subject Classification. Primary 20F10; Secondary 20F65.

The first author acknowledges support from the ANR grant 06-JCJC-0099-01.

The second author's work was supported in part by NSF Grant DMS-0504251.

(C)2008 American Mathematical Society

Reverts to public domain 28 years from publication 
If we consider only splittings over the trivial group, then there is the Grushko decomposition, and it is possible to find this also.

Theorem 1.4. There is an algorithm which takes as input a finite presentation of a group $\Gamma$ which is hyperbolic relative to abelian subgroups, and outputs a presentation for a graph of groups encoding the Grushko decomposition of $\Gamma$, together with an isomorphism to $\Gamma$.

Theorem 1.4 is a major step in the authors' solution in [9] to the Isomorphism Problem for toral relatively hyperbolic groups.

Let us briefly outline the method, and the background. Gerasimov's algorithm for hyperbolic groups is unfortunately still unpublished to this date, and, up to our knowledge, not publicly accessible as an e-print. The results in this paper imply Theorem 1.1 and also extend it to a wide class of groups with the geometry of non-positive curvature. As in Gerasimov's approach, we use connectivity properties of boundaries. In [5], Bowditch associates a natural boundary to every relatively hyperbolic group. This boundary is disconnected if the group admits a splitting over a finite subgroup that is compatible with the relative structure (i.e. in which the parabolic subgroups are elliptic). When the parabolic subgroups are themselves one-ended, this latter requirement is always fulfilled; thus connectivity of the boundary is equivalent to one-endedness in this case. In [1, Bestvina and Mess introduced a condition for (local) connectedness of the boundary of a hyperbolic group, provided this boundary has no global cut point (which Bowditch and Swarup latter proved to never happen for hyperbolic groups). This condition can be stated in the relatively hyperbolic setting as well, but this time, global cut points can occur in the boundary. However, this situation was studied further by Bowditch, who proved, in a quite wide generality, that global cut points are socalled parabolic fixed points and come from peripheral splittings (see Theorem 3.3 below, and the references therein). Bowditch also proved an accessibility theorem for peripheral splittings of relatively hyperbolic groups.

The main idea for detecting the number of ends of a relatively hyperbolic group is to decide whether its boundary is connected. For that we wish to use the condition of Bestvina and Mess, that can be expressed in terms of a finite subset of the group (see Corollary 4.6). There is a one-sided algorithm which will terminate if this condition is satisfied and implies the boundary is connected (see Proposition 5.1). But if there are cut points in the boundary, we want to compute a peripheral splitting so that no vertex group has this problem (by Bowditch's accessibility there is always such a splitting) and test the property vertex by vertex. A first algorithm will enumerate the (peripheral) splittings of the given group, while on each such splitting, another will check whether or not the splitting is nontrivial over a finite group, and if not, a third algorithm will check whether the condition of Bestvina and Mess is fulfilled for each vertex group. This latter algorithm might not terminate on every splitting, but it will terminate at least on a maximal peripheral splitting (the existence of which is guaranteed by Bowditch's accessibility result).

In Section 2 we recall the definition of relatively hyperbolic groups, and some of the geometric and algorithmic properties which we require for this paper. In Section 3 we recall some properties about splittings of relatively hyperbolic groups, due to Bowditch (mostly gathered in Theorem [3.3). The key idea here is that splittings of a relatively hyperbolic group can be detected in terms of cut points on the boundary. In Section 4 we introduce a relatively hyperbolic version of a 
condition of Bestvina and Mess [1], which can be used, if the boundary has no cut points, to discover whether it is connected (Proposition 5.1). Finally, in Section 5 we present the algorithms which prove Theorems 1.21 .3 and 1.4 .

We wish to thank the referee for precise and efficient remarks. These improved the paper, particularly the results in Subsection 2.4.

\section{Preliminaries}

Relatively hyperbolic groups were originally defined by Gromov in his seminal paper [14. An alternative definition was given by Farb [12, who further developed the theory and proved many fundamental results. By now there are many equivalent definitions and characterisations of relatively hyperbolic groups, and there has been a great deal of recent activity.

We will provide a definition of relative hyperbolicity due to the second author and Manning [15] which is suitable for our needs.

2.1. Combinatorial horoballs and the cusped space. In this paragraph, we recall a construction from [15]. For most of this paper, we will only need the 1-skeleton of the cusped space defined in Definition 2.4.

Definition 2.1. Let $\Gamma$ be any 1-complex. The combinatorial horoball based on $\Gamma$, denoted $\mathcal{H}(\Gamma)$, is the 2-complex formed as follows:

- $\mathcal{H}^{(0)}=\Gamma^{(0)} \times(\{0\} \cup \mathbb{N})$.

- $\mathcal{H}^{(1)}$ contains the following three types of edges. The first two types are called horizontal, and the last type is called vertical.

(1) If $e$ is an edge of $\Gamma$ joining $v$ to $w$, then there is a corresponding edge $\bar{e}$ connecting $(v, 0)$ to $(w, 0)$.

(2) If $k>0$ and $0<d_{\Gamma}(v, w) \leq 2^{k}$, then there is a single edge connecting $(v, k)$ to $(w, k)$.

(3) If $k \geq 0$ and $v \in \Gamma^{(0)}$, there is an edge joining $(v, k)$ to $(v, k+1)$.

- $\mathcal{H}^{(2)}$ contains three kinds of 2-cells:

(1) If $\gamma \subset \mathcal{H}^{(1)}$ is a circuit composed of three horizontal edges, then there is a 2-cell (a horizontal triangle) attached along $\gamma$.

(2) If $\gamma \subset \mathcal{H}^{(1)}$ is a circuit composed of two horizontal edges and two vertical edges, then there is a 2-cell (a vertical square) attached along $\gamma$.

(3) If $\gamma \subset \mathcal{H}^{(1)}$ is a circuit composed of three horizontal edges and two vertical ones, then there is a 2 -cell (a vertical pentagon) attached along $\gamma$, unless $\gamma$ is the boundary of the union of a vertical square and a horizontal triangle.

Remark 2.2. As the full subgraph of $\mathcal{H}(\Gamma)$ containing the vertices $\Gamma^{(0)} \times\{0\}$ is isomorphic to $\Gamma$, we may think of $\Gamma$ as a subset of $\mathcal{H}(\Gamma)$.

We also define, for every $k \geq 0$, the $k$-thick part of a combinatorial horoball, denoted $\mathcal{H}(\Gamma)_{k}$, to be the full subcomplex on the set of those vertices of the form $(\gamma, j)$ with $0 \leq j \leq k$.

See [15, Section 3] for a detailed discussion of the geometry of combinatorial horoballs. We recall only the properties which we will need (these statements are [15, Proposition 3.7, Theorem 3.8, Lemma 3.10]). 


\section{Proposition 2.3.}

(1) Let $\Gamma$ be a connected 1-complex so that no edge joins a vertex to itself. Then $\mathcal{H}(\Gamma)$ is simply connected and satisfies a linear combinatorial isoperimetric inequality with constant at most 3.

(2) Let $\Gamma$ be any 1-complex. Then $\mathcal{H}(\Gamma)^{(1)}$ is $\Upsilon$-hyperbolic, and $\Upsilon$ is independent of $\Gamma$.

(3) Let $\Gamma$ be a 1-complex, and $a, b \in \mathcal{H}(\Gamma)^{(0)}$. Then there is a geodesic in $\mathcal{H}(\Gamma)^{(1)}$ joining a to $b$ which consists of at most two vertical segments, one going down (deeper in the horoball) and the other going up, and at most one horizontal segment of length at most 3.

We now proceed to define the cusped space.

Definition 2.4. Let $G$ be a finitely generated group, let $\mathbf{P}=\left\{P_{1}, \ldots, P_{n}\right\}$ be a (finite) family of finitely generated subgroups of $G$, and let $\mathcal{A}$ be a generating set for $G$ so that $P_{i} \cap \mathcal{A}$ generates $P_{i}$ for each $i \in\{1, \ldots, n\}$. For each $i \in\{1, \ldots, n\}$, let $T_{i}$ be a left transversal for $P_{i}$.

For each $i$, and each $t \in T_{i}$, let $\Gamma_{i, t}$ be the full subgraph of the Cayley graph $\Gamma(G, \mathcal{A})$ which contains $t P_{i}$. Each $\Gamma_{i, t}$ is isomorphic to the Cayley graph of $P_{i}$ with respect to the generators $P_{i} \cap \mathcal{A}$. Then we define

$$
X=\Gamma(G, \mathcal{A}) \cup\left(\bigcup\left\{\mathcal{H}\left(\Gamma_{i, t}\right) \mid 1 \leq i \leq n, t \in T_{i}\right\}\right),
$$

where the graphs $\Gamma_{i, t} \subset \Gamma(G, \mathcal{A})$ and $\Gamma_{i, t} \subset \mathcal{H}\left(\Gamma_{i, t}\right)$ are identified as suggested in Remark 2.2.

We call $X$ the cusped space associated to $(G, \mathbf{P}, \mathcal{A})$. We also define the $k$-thick part, $X_{k}$, of the cusped space to be $X_{k}=\Gamma(G, \mathcal{A}) \cup\left(\bigcup\left\{\mathcal{H}\left(\Gamma_{i, t}\right)_{k} \mid 1 \leq i \leq n, t \in T_{i}\right\}\right)$.

2.2. Relatively hyperbolic groups and first algorithms. The cusped space can be used to define relative hyperbolicity:

Definition 2.5. Suppose that $G$ is a finitely generated group and that $\mathbf{P}=$ $\left\{P_{1}, \ldots, P_{n}\right\}$ is a finite collection of finitely generated subgroups of $G$. Suppose further that $\mathcal{A}$ is a finite compatible generating set for $G$.

Then $G$ is hyperbolic relative to $\mathbf{P}$ if the cusped space associated to $(G, \mathbf{P}, \mathcal{A})$ is $\delta$-hyperbolic for some $\delta$.

It is proved in [15, Theorem 3.25] that this definition is equivalent to other standard definitions.

Assumption 2.6. Since the main results of this paper are straightforward in case $G$ is equal to a parabolic subgroup, we will always assume that each parabolic subgroup of $G$ is properly contained in $G$. Since a finite subgroup can be omitted from the list of parabolic subgroups without affecting relative hyperbolicity, we will also assume that parabolic subgroups are infinite.

Definition 2.7. Let $G$ be a relatively hyperbolic group, with associated cusped space $X$. The boundary of $G$, denoted $\partial G$, is the Gromov boundary of the space $X$ (defined as usual by equivalence classes of quasi-geodesic rays).

It follows from [5] Sections 6,9] that the above definition of $\partial G$ agrees with Bowditch's definition (the analysis in [5, Section 6] applies to our cusped space 
$X$ ). In particular, the results of Bowditch quoted in Section 3 below apply to the boundary defined in Definition 2.7.

Let us now recall the main result of 8 .

Theorem 2.8 ([8, Theorems 0.2 and 0.1]). There is an algorithm that, given a finite presentation of a group $G$, terminates if and only if $G$ is hyperbolic relative to abelian subgroups. In case it terminates, it provides a finite presentation of each of the parabolic subgroups (up to conjugacy) in terms of the given generators of $G$, together with a constant for a linear relative isoperimetric inequality (or equivalently a linear combinatorial isoperimetric inequality of the coned-off Cayley complex; see [15, Section 2]).

Remark 2.9. Given a finite presentation of an abelian group, it is straightforward to determine if the group is finite or not. Therefore, it is no trouble to make the Assumption 2.6 that all parabolic subgroups are infinite.

If a parabolic subgroup is virtually cyclic, we can exclude it from the list of parabolic subgroups without affecting relative hyperbolicity. Since we can determine if an abelian group is virtually cyclic from a finite presentation, we can also assume that all parabolic subgroups are not virtually cyclic.

We require one further result which will allow us to effectively compute a value of $\delta$ for which the cusped space is $\delta$-hyperbolic.

Theorem 2.10 ([15, Theorem 3.24]). Suppose that $G, \mathbf{P}$ and $\mathcal{A}$ are as in Definition 2.5 and that $G$ is hyperbolic relative to $\mathbf{P}$. If the coned-off Cayley complex of $G$ associated to $G, \mathbf{P}, \mathcal{A}$ satisfies a linear combinatorial isoperimetric inequality with constant $K$, then the cusped space associated to $G, \mathbf{P}, \mathcal{A}$ satisfies a linear isoperimetric inequality with constant $K_{1}=3 K(2 K+1)$.

The space $X$ is the cusped space from Definition 2.4. We can compute $\delta$ using Theorems 2.8 and 2.10 ,

Let $v_{0}$ be the natural base point of $X$, corresponding to the identity element of $G$ which is a vertex of the Cayley graph of $G$, which is embedded in the cusped space $X$.

Balls of $X$ can be computed by using a solution to the word problem in $G$ (and the fact that we know we have a compatible generating set, and we know which of our generators lie in which parabolic subgroups - this uses the fact that the parabolic subgroups are abelian).

Lemma 2.11. For every point $x \in X$ there is a geodesic ray which starts at $v_{0}$ and passes within $3 \delta$ of $x$.

Proof. Suppose that $x \in X$ has the form $(\gamma, k)$, where $\gamma \in t_{j} P_{i}$, for some parabolic subgroup $P_{i}$ and some transversal element $t_{i}$. (Note that in case $k=0$, there may be more than one such pair $\left(P_{i}, t_{j}\right)$. This is unimportant in the following argument.)

Let $\alpha \in \partial G$ be the point in the boundary fixed by $t_{j} P_{i} t_{j}^{-1}$, corresponding to the horoball built from $t_{j} P_{i}$. Since $P_{i}$ is a proper subgroup of $G$, there exist $\beta \in \partial G$ so that $\beta$ is the fixed point of some $t_{k} P_{i} t_{k}^{-1}$, and $t_{k} P_{i} \neq t_{j} P_{i}$.

Let $p$ be the path which consists of a shortest path between the horoballs $t_{j} P_{i}$ and $t_{k} P_{i}$, and two vertical paths to $\alpha$ and $\beta$. It is easy to see that $p$ is a bi-infinite geodesic path. By applying an element of $t_{j} P_{i} t_{j}^{-1}$ to the path $p$, we may assume that $p$ passes through $x$. 
According to [15, Lemma 2.11] (which is a simple exercise), a geodesic triangle in a $\delta$-hyperbolic space with some or all vertices ideal is $3 \delta$-slim. Consider a partially ideal triangle with vertices $v_{0}, \alpha, \beta$, where the path between $\alpha$ and $\beta$ is $p$. Then $x$ lies within at most $3 \delta$ from one of the edges $\left[v_{0}, \alpha\right],\left[v_{0}, \beta\right]$, as required.

2.3. Constants. We choose $\delta$ to be a positive integer so that $X$ is $\delta$-hyperbolic.

Let $C=3 \delta$, so by Lemma 2.11 any point in $X$ is at distance at most $C$ from a geodesic ray starting at $v_{0}$.

Let us define $M=6(C+45 \delta)+2 \delta+3$, and $k=2 M$, and recall that $X_{k}$ is the $k$-thick part of the cusped space. Let $K=3\left(2^{2 M+3}\right)+M+3$. Finally, let $R(n)=4(n+M)+3 k+50 \delta+3$.

2.4. Two properties of horoballs. Recall that in any metric space $(X, d)$ the Gromov product of points $x$ and $y$ with respect to a third point $z$ is

$$
(x \cdot y)_{z}=\frac{1}{2}(d(x, z)+d(y, z)-d(x, y)) .
$$

Given a comparison tripod $Y_{x y z}$ for $x, y, z$ (where the vertices of the tripod are $\bar{x}, \bar{y}, \bar{z})$, the distance from $\bar{z}$ to the centre of the tripod is $(x \cdot y)_{z}$. In particular, if $z$ lies on a geodesic between $x$ and $y$, then $(x \cdot y)_{z}=0$.

A definition of $\delta$-hyperbolicity is that for all $w, x, y, z \in X$ we have

$$
(x \cdot y)_{w} \geq \min \left\{(x \cdot z)_{w},(y \cdot z)_{w}\right\}-\delta .
$$

(Because the constants for translating between the various definitions of $\delta$-hyperbolicity are explicit, we assume that the above equation holds as well as triangles being $\delta$-thin, and $\delta$-slim. See [6, III.H] for more details.)

Lemma 2.12. Let $H_{k}$ be a horoball at depth $k>0, p_{0} \in H_{k}$, and $p_{1} \in H_{k-1} \backslash H_{k}$. Then, there exists $p_{2} \in H_{k-1} \backslash H_{k}$ such that $d\left(p_{0}, p_{2}\right) \leq d\left(p_{0}, p_{1}\right)+3$, and $\left(p_{1} \cdot p_{2}\right)_{p_{0}} \leq$ 3 .

Proof. Suppose that the horoball is built on the graph $\Gamma$, so its vertices are labelled by $(\gamma, i)$ where $\gamma \in \Gamma^{(0)}$ and $i \geq k-1$. Note that we are assuming that $\Gamma$ has infinite diameter.

We consider the geodesic $\gamma$ between $p_{0}$ and $p_{1}$. By Proposition 2.3. (3), we may assume that $\gamma$ either consists of a single vertical segment, or else at most two vertical segments and a single horizontal segment of length at most 3 . We distinguish three cases: where $\gamma$ contains two vertical segments (and one horizontal segment); where $\gamma$ is entirely vertical; and where $\gamma$ contains a single vertical segment and a horizontal segment.

Suppose first that this geodesic contains a horizontal segment and two vertical segments, and suppose that $p_{0}=(\gamma, i)$. Then the path formed by concatenating the geodesic from $p_{1}$ to $p_{0}$ and the vertical path from $p_{0}$ to $(\gamma, k-1)$ is a geodesic, and $\left(p_{1} \cdot(\gamma, i)\right)_{p_{0}} \leq \frac{1}{2} \delta$, so we may take $p_{2}=(\gamma, k-1)$. In this case certainly we have $d\left(p_{0}, p_{2}\right) \leq d\left(p_{0}, p_{1}\right)$. Note also that since $p_{0}$ lies on a geodesic from $p_{1}$ to $p_{2}$ we must have $\left(p_{1} \cdot p_{2}\right)_{p_{0}}=0$.

Next suppose that the geodesic from $p_{1}$ to $p_{0}$ is vertical, so $p_{1}=(\gamma, k-1)$ and $p_{0}=(\gamma, i)$ for some $\gamma \in \Gamma^{(0)}$ and some $i>k-1$. Note that if $d\left(p_{0}, p_{1}\right) \leq \delta$, then we can take $p_{2}=p_{1}$ and the lemma is trivial. Thus we may suppose that $i-k-1>\delta$.

Since the graph $\Gamma$ has infinite diameter, there is a vertex $\gamma_{0} \in \Gamma^{(0)}$ so that $d_{\Gamma}\left(\gamma, \gamma_{0}\right)=3\left(2^{i}\right)$. Thus the distance between $(\gamma, i)$ and $\left(\gamma_{0}, i\right)$ at depth $i$ is 3 , 
and there is a horizontal path between them which is a geodesic in $H_{k}$. The path between $(\gamma, k-1)$ and $\left(\gamma_{0}, k-1\right)$ which consists of the vertical paths between $(\gamma, k-1)$ and $(\gamma, i)$ and between $\left(\gamma_{0}, k-1\right)$ and $(\gamma, k-1)$ and the horizontal path between $(\gamma, i)$ and $\left(\gamma_{0}, i\right)$ is a geodesic in $H_{k}$. Let $p_{2}=(\gamma, k-1)$. Clearly $d\left(p_{0}, p_{2}\right)=d\left(p_{0}, p_{1}\right)+3$, and it is not hard to see that $\left(p_{1} \cdot p_{2}\right)_{p_{0}}=0$ (since again $p_{0}$ lies on a geodesic between $p_{1}$ and $p_{2}$ ).

Finally, suppose that the geodesic from $p_{1}$ to $p_{0}$ contains a single vertical segment and a horizontal segment. In this case the geometry of the graph on which the horoball is based may prevent us from finding a point $p_{2} \in H_{k-1} \backslash H_{k}$ so that $p_{0}$ lies on a geodesic from $p_{1}$ to $p_{2}$. However, let $x$ be the point at the end of the vertical segment in the geodesic from $p_{1}$ to $p_{0}$. Then $d\left(x, p_{0}\right) \leq 3$ and $d\left(p_{0}, p_{1}\right)=$ $d\left(p_{0}, x\right)+d\left(x, p_{1}\right)$. The second case above finds a point $p_{2}$ so that $x$ lies on the geodesic from $p_{1}$ to $p_{2}$ and $d\left(x, p_{2}\right)=d\left(x, p_{1}\right)+3$. Since $x$ lies on the geodesic from $p_{1}$ to $p_{2}$ a simple calculation then shows that $\left(p_{1} \cdot p_{2}\right)_{p_{0}} \leq d\left(x, p_{0}\right) \leq 3$. Also, $d\left(p_{0}, p_{2}\right) \leq d\left(p_{0}, x\right)+d\left(x, p_{2}\right)=d\left(p_{0}, x\right)+d\left(x, p_{1}\right)+3=d\left(p_{0}, p_{1}\right)+3$, as required.

This finishes the proof in all cases.

Definition 2.13. Suppose that $P$ is a finitely generated abelian group. A finite generating set $A$ for $P$ is called sensible if it can be partitioned into two subsets $A=A_{1} \sqcup A_{2}$ where $A_{1}$ is a basis for a free abelian group and $A_{2}$ is a generating set for a finite abelian group $P_{f}$, and $\left|A_{2}\right|$ is minimal amongst all generating sets of $P_{f}$.

Remark 2.14. The algorithm from 8 which finds the relatively hyperbolic structure for a relatively hyperbolic group with abelian parabolic subgroups finds a sensible generating set for the parabolic subgroups. Therefore, whenever $G$ is assumed to have abelian parabolic subgroups, we will assume that the generating set $\mathcal{A}$ is such that the intersection of $\mathcal{A}$ with any element of $\mathbf{P}$ is sensible.

Lemma 2.15. Suppose that $A$ is a sensible generating set for an infinite abelian group $P$ which is not virtually cyclic. For any $r \geq 1$, if $a, b \in P$ are at distance at least $r$ from 1 , then there is a path of length at most $3 d(a, b)$ from a to $b$ which does not intersect the ball of radius $r-1$ about 1 (all distances are measured with respect to the word metric coming from the sensible generating set).

Proof. Let $A=A_{1} \sqcup A_{2}$, where $A_{1}$ generates a free abelian subgroup $P_{1}$ of $P$, and $A_{2}$ generates a finite group. Since $P$ is not virtually cyclic, $P_{1}$ has rank at least 2 .

If $d(a, b) \leq 1$, there is nothing to prove.

Let us write $a=\left(a_{1}, a_{2}\right)$, with $a_{i} \in\left\langle A_{i}\right\rangle$. and $b=\left(b_{1}, b_{2}\right)$ similarly. Let us consider the coordinates of $a_{1}=\left(x_{1}, \ldots, x_{m}\right)$ and $b_{1}=\left(y_{1}, \ldots, y_{m}\right)$ in the basis $A_{1}$ (we choose additive notation). If two coordinates corresponding to a basis element $e \in A_{1}$ are of the same sign (assume it is positive, up to changing $e$ into $-e$ ), then $e+a$ and $e+b$ are both at distance at least $r+1$ from 1 . If $\sigma$ is a geodesic segment between $a$ and $b$, then $e+\sigma$ has its extremal edges outside the ball of radius $r$ about 1. Considering the neighbors of $e+a$ and $e+b$ on $e+\sigma$, we get two points $a^{\prime}$ and $b^{\prime}$ satisfying the assumption of the lemma, with $d\left(a^{\prime}, b^{\prime}\right)=d(a, b)-2$, and it takes two paths of length 2 to reach them from $a$ and $b$ outside the ball of radius $r-1$ about 1 .

Thus by iterating this process, we can reduce the case to that where all coordinates of $a_{1}=\left(x_{1}, \ldots, x_{m}\right)$ have opposite signs from those of $b_{1}=\left(y_{1}, \ldots, y_{m}\right)$. 
Let us write $\left\langle A_{2}\right\rangle$ as a direct product of cyclic groups, and $a_{2}=\left(\alpha_{1}, \ldots, \alpha_{k}\right)$ and $b_{2}=\left(\beta_{1}, \ldots, \beta_{k}\right)$ in this product. If, in the cyclic component, 0 is not on a geodesic between $\alpha_{i}$ and $\beta_{i}$, and assuming that $a$ is closer to 1 than $b$, then there is a path of length $\left\|\alpha_{i}-\beta_{i}\right\|$ between $a$ and $a^{\prime}$, not approaching 1 , such that the coordinate of $a^{\prime}$ along this cyclic factor is that of $b$. The distance to $b$ has been reduced by the same amount; thus, we can reduce to the case where, in $\left\langle A_{2}\right\rangle$, a geodesic between $a_{2}$ and $b_{2}$ goes through 0 , that is, $\left\|a_{2}-b_{2}\right\|=\left\|a_{2}\right\|+\left\|b_{2}\right\|$.

Up to changing some elements $e$ of the basis $A_{1}$ into $-e$, we can assume that all coordinates of $a_{1}$ are negative, and all those of $b_{1}$ are positive. Then, by adding $(0, \ldots, 0,-1) \in\left\langle A_{1}\right\rangle$, then $(1,0,0, \ldots, 0) \in\left\langle A_{1}\right\rangle$ to $a$, exactly $\left|x_{1}\right|$ times, one defines a path of length $2\left|x_{1}\right|$, outside the ball of radius $r-1$, to a point with 0 first coordinate. Thus, we can find a path from $a$ to $\left(\left(0, \ldots,-\sum_{1}^{m}\left|x_{i}\right|-\left\|a_{2}\right\|\right), 0\right)$ of length at most $2 \sum_{1}^{(m-1)}\left|x_{i}\right|$, outside the ball of radius $(r-1)$. Similarily, one can find a path from $b$ to $\left(\left(\sum_{1}^{m} y_{i}+\left\|b_{2}\right\|, 0, \ldots, 0\right), 0\right)$, of length at most $2 \sum_{2}^{m} y_{i}+$ $2\left\|b_{2}\right\|$, outside the forbidden ball. These two paths have total length at most $2 \sum_{1}^{m}\left(y_{i}-x_{i}\right)+2\left\|a_{2}-b_{2}\right\|$ (recall $x_{i}<0$, and $\left.\left\|a_{2}-b_{2}\right\|=\left\|a_{2}\right\|+\left\|b_{2}\right\|\right)$, thus at most $2 d(a, b)$. Notice now that there is a path between $\left(\left(0, \ldots,-\sum_{1}^{m}\left|x_{i}\right|-\left\|a_{2}\right\|\right), 0\right)$ and $\left(\left(\sum_{1}^{m} y_{i}+\left\|b_{2}\right\|, 0, \ldots, 0\right), 0\right)$, via $\left(\left(\sum_{1}^{m} y_{i}+\left\|b_{2}\right\|, 0, \ldots, 0,-\sum_{1}^{m}\left|x_{i}\right|-\left\|a_{2}\right\|\right), 0\right)$, and of length $\sum_{1}^{m} y_{i}-x_{i}+\left\|a_{2}\right\|+\left\|b_{2}\right\|=d(a, b)$. This path does not get closer to 1 than its end points. To summarise, there is a path of length at most $3 d(a, b)$ from $a$ to $b$ outside the forbidden ball.

Recall that we defined $K=3\left(2^{2 M+3}\right)+M+3$.

Lemma 2.16. Assume that the parabolic subgroups of $G$ are abelian, and that the intersection of $\mathcal{A}$ with each element of $\mathbf{P}$ forms a sensible generating set.

Let $H_{k}$ be a horoball at depth $k>0$, and $x, y$ in $H_{k}$ be such that $d(x, y) \leq M$, and $\left|d\left(x, v_{0}\right)-d\left(y, v_{0}\right)\right| \leq 20 \delta$.

Then, there exists a path between $x$ and $y$ in $H_{k}$ which is of length at most $K$ and which does not intersect the ball of radius $m=\min \left\{d\left(v_{0}, x\right), d\left(v_{0}, y\right)\right\}$ about $v_{0}$.

Proof. Note that in the proof below we appeal to Lemma 2.15. If the parabolic subgroup associated to $H_{k}$ is virtually cyclic, then Lemma 2.15 does not apply. However, in this case the horoball is quasi-isometric to $\mathbb{H}^{2}$. Using this observation, a proof of Lemma 2.16 in this case can be obtained without trouble. Therefore, we assume that the parabolic subgroup is not virtually cyclic.

Let $\sigma_{1}$ be a geodesic from $v_{0}$ to $x$ whose intersection with $H_{k}$ satisfies the conclusions of Proposition 2.3(3), and $\sigma_{2}$ such a geodesic from $v_{0}$ to $y$. Since, for all $i$, $H_{i}$ is convex in $X$, the points at which $\sigma_{1}$ and $\sigma_{2}$ first intersect $H_{1}$ are a distance at most $2 \delta$ apart, and since $k>1+\log _{2}(2 \delta)$, the points at which $\sigma_{1}$ and $\sigma_{2}$ first intersect $H_{k}$ are a distance at most 1 apart.

The paths $\sigma_{1}$ and $\sigma_{2}$ then travel vertically at a distance at most 1 apart. Then one of the paths turns horizontal, and goes back up, whilst the other possibly goes deeper, before possibly turning and going up also. If both paths contain only a single vertical segment, the lemma is straightforward: suppose $x$ is the higher of $x$ and $y$. Then go directly downwards from $x$ to the depth of $y$, and then apply Lemma 2.15. Thus we can assume that $\sigma_{1}$ contains two vertical segments.

Suppose (by relabelling $x$ and $y$ if necessary) that $x$ lies no deeper than $y$, and that the depth of $x$ is $i$. Since $x$ and $y$ lie no more than $M$ apart in $X$, we may 
append a path $\gamma$ to the end of $\sigma_{2}$ so that $\sigma_{2} \cup \gamma$ is a geodesic and so that $\gamma$ :

(1) lies outside of the ball of radius $m$ about $v_{0}$;

(2) is entirely vertical except possibly for a horizontal segment of length at most 3 ;

(3) has length at most $M+3$; and

(4) has end $y^{\prime}$ (at the other end of $y$ ) which is at depth $i$ (the same as $x$ ) and is at distance at most $2 M+3$ from $x$ in $X$.

We will find a path from $y^{\prime}$ to $x$ of bounded length which stays outside of the ball of radius $m$ about $v_{0}$. Let $\sigma_{2}^{\prime}$ be the appended geodesic $\sigma_{2} \cup \gamma$.

Let $d_{i}$ denote the distance at depth $i$. Since the distance in $X$ between $x$ and $y^{\prime}$ is at most $2 M+3$, we have $d_{i}\left(x, y^{\prime}\right) \leq 2^{2 M+3}$.

The intersection of level $i$ with $\sigma_{1}$ consists of two points, $x$ and another point $p_{1}$. The intersection of $\sigma_{2}^{\prime}$ with level $i$ consists of points $y^{\prime}$ and $p_{2}$. Note that, since $i>k$, as noted before, one has $d\left(p_{1}, p_{2}\right) \leq 1$; hence $d_{i}\left(p_{1}, p_{2}\right) \leq 1$.

Now, the part of $H_{k}$ at level $i$ is a Cayley graph of a finitely generated abelian group $P$, where $P \in \mathbf{P}$. This Cayley graph is with respect to a generating set consisting of all elements of length at most $2^{i}$ in a sensible generating set of $P$. Let $L_{i}$ be this Cayley graph at depth $i$ in $H_{k}$. By Lemma 2.15, for any points $a$ and $b$ in $L_{1}$ which lie at distance at least $r$ from 1 , there is a path of length at most $3 d_{1}(a, b)$ between $a$ and $b$ which does not intersect the ball of radius $r-1$ about 1 . Since the distance in $L_{i}$ between points $u=\left(u_{0}, i\right), v=\left(v_{0}, i\right) \in L_{i}$ is $\left\lfloor 2^{-i} d_{1}\left(u_{0}, v_{0}\right)\right\rfloor$, it is easy to see that Lemma 2.15 holds for $L_{i}$ also.

Let $\sigma$ be a path in $L_{i}$ between $x$ and $y^{\prime}$ which does not come closer to $p_{1}$ and $p_{2}$ than $x$ or $y^{\prime}$, and is of minimal length with respect to this restriction. By the remark above, the length of $\sigma$ is at most $3 d_{i}\left(x, y^{\prime}\right) \leq 3\left(2^{2 M+3}\right)$.

In conclusion, there is a path between $x$ and $y^{\prime}$ of the right kind of length at most $3\left(2^{2 M+3}\right)$, and the path between $y$ and $y^{\prime}$ has length at most $M+3$. This finishes the proof of the lemma.

Remark 2.17. There are versions of Lemma 2.16 with similar conclusions and much weaker assumptions on the parabolic subgroups. However, we were unable to prove a result with no assumptions on the parabolic subgroups, and different types of paths of different lengths and with slightly different conclusions are needed for different types of parabolic subgroups. Thus, we decided to present a simple version which suffices for the needs of this paper.

\section{Splittings}

In this section we gather some results due to B. Bowditch about splittings. We first need a characterisation of connectedness of the boundary.

Proposition 3.1 ([5, Propositions 10.1-3]). Let $(G, \mathbf{P})$ be a relatively hyperbolic group. Its boundary $\partial G$ is disconnected if and only if $G$ splits nontrivially over a finite group relative to $\mathbf{P}$. In this case, every vertex group is hyperbolic relative to the parabolic subgroups it contains.

In particular, if the parabolic subgroups of $G$ are all one-ended, then $G$ is oneended if and only if $\partial G$ is connected.

In the above result, a splitting is relative to $\mathbf{P}$ if each element of $\mathbf{P}$ is elliptic in the splitting. 
Then we need to characterise the presence of global cut points. For that, we recall the notion of peripheral splitting.

Definition $3.2(4)$. Let $(G, \mathbf{P})$ be a relatively hyperbolic group. A peripheral splitting of $G$ is a representation of $G$ as a finite bipartite graph of groups where $\mathbf{P}$ consists precisely of the (conjugacy classes of) vertex groups of one colour. A peripheral splitting is a refinement of another if there is a colour preserving folding of the first into the second.

Theorem 3.3 (B. Bowditch). Let $(G, \mathbf{P})$ be a relatively hyperbolic group. Assume that $\partial G$ is connected.

(1) [2, Theorem 0.2] If every maximal parabolic subgroup of $G$ is (one or two)ended, finitely presented, and without infinite torsion subgroup, then, every global cut point of $\partial G$ is a parabolic fixed point.

(2) [3, Theorem 1.2] If there is a global cut point that is a parabolic point, then $G$ admits a proper peripheral splitting.

(3) [4, Theorem 1.2] If $G$ admits a proper peripheral splitting, then $\partial G$ admits a global cut point.

It is established in [4, Theorem 1.3] that the vertex groups of peripheral splittings are hyperbolic relative to the trace of the parabolic subgroups of $G$ on them, and that if $\partial G$ is connected, then any nonperipheral vertex group also has connected boundary and is hyperbolic relative to its adjacent edge groups.

We also need an accessibility result.

Theorem 3.4 ([4, Theorem 1.4]). Suppose that $G$ is relatively hyperbolic with connected boundary. Then $G$ admits a (possibly trivial) peripheral splitting which is maximal in the sense that it is not a refinement of any other peripheral splitting.

The boundaries of the components of such a maximal splitting do not contain any global cut point.

We will recognise when the boundary is connected without global cut point, on the one hand, and when the group admits nontrivial splittings on the other.

\section{On a condition of M. Bestvina and G. Mess} 2 .

Let $G$ be a relatively hyperbolic group, and $X$ the cusped space defined in Section

Let us introduce a slight variation of the property $\ddagger$ of Bestvina and Mess, defined in $[1$.

Given $\epsilon \geq 0$, and two points $x, y$ in $X$, we say that $x$ and $y$ satisfy $\star_{\epsilon}$ if

$$
\star_{\epsilon}: \quad\left|d\left(v_{0}, x\right)-d\left(v_{0}, y\right)\right| \leq \epsilon \text { and } d(x, y) \leq M .
$$

Given an integer $n$, we say that two points $x, y$ satisfying $\star_{\epsilon}$ satisfy $\ddagger(\epsilon, n)(x, y)$ if there is a path of length at most $n$ from $x$ to $y$, in the complement in $X$ of the ball $B_{v_{0}}(m-C-45 \delta+3 \epsilon)$, where $m=\min \left\{d\left(v_{0}, x\right), d\left(v_{0}, y\right)\right\}$.

First one can reproduce verbatim the argument of Bestvina and Mess (we refer the reader to [1, Proposition 3.2]) to get the following property. 
Lemma 4.1. If there exists an $n$ such that for all $x, y$ in $X$ satisfying $\star_{0}$, the property $\ddagger(0, n)(x, y)$ holds, then $\partial G$ is connected.

Note that if $\ddagger(0, n)(x, y)$ holds, then $\ddagger(0, m)(x, y)$ holds for all $m \geq n$ also. Following another argument of Bestvina and Mess ([1, Proposition 3.3]), we also obtain (recall that we defined the constant $k$ in Subsection 2.3):

Lemma 4.2. If the boundary $\partial G$ is connected, and has no global cut point, then there is $n$ such that $\ddagger(10 \delta, n)(x, y)$ holds for all $x, y$ in $X_{k}$.

Proof. We prove the contrapositive. If for each $n$ there are $x_{n}, y_{n}$ so that $\ddagger(10 \delta, n)\left(x_{n}, y_{n}\right)$ is false, then we will find a point $\xi$ in $\partial G$ such that $\partial G \backslash\{\xi\}$ is disconnected.

Thus, let us assume that $\ddagger(10 \delta, n)\left(x_{n}, y_{n}\right)$ is false for a sequence of vertices $x_{n}, y_{n}$ in $X_{k}$. Let $r_{n}$ and $s_{n}$ be geodesic rays from $v_{0}$ that pass at a distance at most $C$ from $x_{n}$ and $y_{n}$, respectively. Their points at infinity are denoted by $\alpha_{n}$ and $\beta_{n}$.

Since there are finitely many orbits of vertices in $X_{k}$, one can find a sequence of elements $\gamma_{n}$ such that, after extraction of a subsequence, $\gamma_{n} x_{n}$ is constant, say equal to $x$. Since $y_{n}$ is at bounded distance of $x_{n}$, and the space $X$ is proper, we can assume that $\gamma_{n} y_{n}$ is constant, say equal to $y$, with $d(x, y) \leq M$. Without loss of generality, one can assume that $\min \left\{d\left(v_{0}, x_{n}\right), d\left(v_{0}, y_{n}\right)\right\}=d\left(v_{0}, x_{n}\right)$, for all $n$ in the extracted subsequence. We also extract in order that $\gamma_{n} \alpha_{n} \rightarrow \alpha$, and $\gamma_{n} \beta_{n} \rightarrow \beta$ in $\partial X, \gamma_{n} v_{0} \rightarrow \xi \in \partial G,\left[x, \gamma_{n} v_{0}\right] \rightarrow[x, \xi)=\rho$ and $r_{n} \rightarrow r$, and $s_{n} \rightarrow s$, bi-infinite geodesics from $\xi$ to $\alpha$, and $\beta$ (it is easy to check that $\xi$ is different from $\alpha$ and $\beta$ ).

We consider then $B_{n}=\gamma_{n} B\left(v_{0}, d\left(v_{0}, x_{n}\right)-15 \delta-C\right)$.

Let $B=\bigcup_{\mathbb{N}} B_{n}$. Clearly $\xi \in \bar{B} \cap \partial X$ for the usual topology on $X \cup \partial X$. One has furthermore $\{\xi\}=\bar{B} \cap \partial X$. For, if $\zeta \in \partial X \backslash\{\xi\}$, then, letting $\left\{f_{n}\right\}$ be a sequence of points in $B_{n}$ going to $\zeta$, by hyperbolicity in the pentagons $\left(x, \xi, \gamma_{n} v_{0}, f_{n}, \zeta\right)$, there is $N>0$ such that if $n$ is large enough, any path from $\gamma_{n} v_{0}$ to $f_{n}$ passes at a distance at most $N$ from $x$. This provides $d\left(\gamma_{n} v_{0}, f_{n}\right)>2\left(d\left(v_{0}, x_{n}\right)-15 \delta-C\right)$, for $n$ large enough, contradicting the assumption that both are in $B_{n}$.

The space $X \backslash B$ is disconnected. Indeed, $x$ and $y$ are in $X \backslash B$, and if there is a path $\lambda$ in $X \backslash B$, from $x$ to $y$, then, for $n>\operatorname{length}(\lambda)$, translating by $\gamma_{n}^{-1}$, we see that $\ddagger(10 \delta, n)$ is true for $\left(x_{n}, y_{n}\right)$, contradicting the assumption on $x_{n}, y_{n}$.

The distance between $x$ and $r$ is at most $C$, and that between $x$ and $B$ is $15 \delta+C$. Therefore the ray $r$ enters the component of $x$ in $X \backslash B$. Similarly, the ray $s$ enters the component of $y$ in $X \backslash B$.

For a component $A$ of $X \backslash B$, let us consider $\mathcal{O}(A) \subset \partial X \backslash\{\xi\}$, the set of the points $\zeta$ such that a bi-infinite geodesic $(\xi, \zeta)$ enters $A$.

The set $\mathcal{O}(A)$ is open: indeed, if $\zeta$ is in it, a neighborhood of $\zeta$ consists of points $\eta$ such that $(\xi, \eta)$ remains at a distance $10 \delta$ from $(\xi, \zeta)$, from $\xi$ until some point at distance at least $20 \delta$ from $B$, and therefore these geodesics must enter the same component of $X \backslash B$ as $(\xi, \zeta)$.

If $A^{\prime} \neq A, \mathcal{O}(A)$ and $\mathcal{O}\left(A^{\prime}\right)$ are disjoint, because if a point $\zeta$ is in the intersection, one geodesic $(\xi, \zeta)$ enters $A$, although another enters $A^{\prime}$, but both remain at a distance $2 \delta$, thus providing paths between $A$ and $A^{\prime}$ that avoid $B$ (contradicting that $A \neq A^{\prime}$ ).

Finally there are at least two nonempty such open sets (one containing $\alpha$, and one containing $\beta$ ), and the union of them covers $\partial X \backslash\{\xi\}$. All this proves that $\partial G \backslash\{\xi\}$ is disconnected. 
We now want to reduce $\ddagger(0, n)$ on $X$, to $\ddagger(10 \delta, n)$ on some finite set. We begin with two lemmata, dealing with the $k$-thick part $X_{k}$. Recall the definition of $R(n)$ from Subsection 2.3 .

Lemma 4.3. For all $x$ in $X_{k}$ with $d\left(x, v_{0}\right) \geq R(n)$, if $p$ is a point on a geodesic $\left[v_{0}, x\right]$ at a distance $2(n+M)$ from $x$, then there exists an element $\gamma$ such that $\left(\gamma v_{0} \cdot x\right)_{p} \leq \delta$, and $d\left(x, \gamma v_{0}\right) \leq R(n)-50 \delta$.

Notice that this is straightforward for hyperbolic groups, since in the Cayley graph, one can always choose $\gamma v_{0}=p$.

Proof. Let us consider the subsegment $\left[p^{\prime}, p\right]$ of $\left[v_{0}, p\right]$ and of length $4 k$ (it exists since $\left.d\left(v_{0}, x\right) \geq R(n)\right)$. If the distance between $p^{\prime}$ and $G v_{0}$ is at most $3 k$, then the element $\gamma$ such that $\gamma v_{0}$ is closest to $p^{\prime}$ is suitable.

If now $p^{\prime}$ is at a distance at least $3 k$ from $G v_{0}$, then it is not in $X_{k}$. Since $x \in X_{k}$, there is a first point $p^{\prime \prime}$ on $\left[p^{\prime}, x\right]$ that is in $X_{k}$.

By assumption on $p^{\prime}$, one has $d\left(p^{\prime}, p^{\prime \prime}\right) \geq 2 k$. Moreover, since the horoballs at depth $k$ are separated, the segment $\left[p^{\prime}, p^{\prime \prime}\right]$ remains in a horoball. By Lemma 2.12, there is another point $q$ in $X_{k}$ with $d\left(p^{\prime}, q\right) \leq d\left(p^{\prime}, p^{\prime \prime}\right)+3$ and such that $\left(q \cdot p^{\prime \prime}\right)_{p^{\prime}} \leq 3$

Since $q \in X_{k}$, there exists $\gamma \in G$ such that $d\left(\gamma v_{0}, q\right) \leq k$. Recall that $d\left(p^{\prime}, \gamma v_{0}\right) \geq$ $3 k$; hence $d\left(p^{\prime}, q\right) \geq 2 k$. Therefore, for this element,

$$
\left(\gamma v_{0} \cdot q\right)_{p^{\prime}} \geq\left(d\left(p^{\prime}, q\right)+d\left(p^{\prime}, \gamma v_{0}\right)-k\right) / 2 \geq 2 k,
$$

and by hyperbolicity, it follows that $\left(\gamma v_{0} \cdot p^{\prime \prime}\right)_{p^{\prime}} \leq \delta+3$. Since $\left(p \cdot p^{\prime \prime}\right)_{p^{\prime}}=d\left(p^{\prime}, p^{\prime \prime}\right) \geq$ $2 k$, one has $\left(\gamma v_{0} \cdot p\right)_{p^{\prime}} \leq 2 \delta+3$. Therefore, $\left(\gamma v_{0} \cdot p^{\prime}\right)_{p} \geq d\left(p, p^{\prime}\right)-(2 \delta+3)>\delta$. Since $\left(x \cdot p^{\prime}\right)_{p}=0$, by hyperbolicity, $\left(\gamma v_{0} \cdot x\right)_{p} \leq \delta$.

Moreover, one has $d\left(x, \gamma v_{0}\right) \leq d\left(x, p^{\prime}\right)+d\left(p^{\prime}, q\right)+d\left(q, \gamma v_{0}\right)$. Recall that $d\left(p^{\prime}, q\right) \leq$ $d\left(p^{\prime}, p^{\prime \prime}\right)+3 \leq d\left(p^{\prime}, x\right)+3$, and that $d\left(q, \gamma v_{0}\right) \leq k$. Since $d\left(p^{\prime}, x\right) \leq d(p, x)+k$, one has $d\left(x, \gamma v_{0}\right) \leq 2 d(x, p)+6 k+3$, that is, at most $4(n+M)+3 k+3 \leq R(n)-50 \delta$. Therefore, $\gamma v_{0}$ is suitable.

Lemma 4.4. Assume that, for some $n, \ddagger(10 \delta, n)(x, y)$ holds for all pairs of vertices $(x, y)$ in $B_{v_{0}}(R(n))$ satisfying $\star_{10 \delta}$.

Let $x, y \in X_{k}$, satisfying $\star_{0}$, and $d\left(v_{0}, x\right) \geq R(n)$. Let $p$ be on a geodesic segment $\left[v_{0}, x\right]$ at distance $2(n+M)$ from $x$, as in the previous lemma. Then the pair $(x, y)$ satisfies $\ddagger(0, n)$.

Proof. By Lemma 4.3, there exists $\gamma \in G$ such that $\left(\gamma v_{0} \cdot x\right)_{p} \leq \delta$ and $d\left(\gamma v_{0}, x\right) \leq$ $R(n)-50 \delta$. Let us first prove that $\left(\gamma^{-1} x, \gamma^{-1} y\right)$ satisfies $\star_{10 \delta}$.

By hyperbolicity, $\min \left\{\left(\gamma v_{0} \cdot y\right)_{p},(x \cdot y)_{p}\right\} \leq\left(\gamma v_{0} \cdot x\right)_{p}+\delta \leq 2 \delta$. Since $(x \cdot y)_{p} \geq M+$ $2 n$, it follows that $\left(\gamma v_{0} \cdot y\right)_{p} \leq 2 \delta$, and therefore, $d\left(\gamma v_{0}, y\right) \geq d\left(\gamma v_{0}, p\right)+d(p, y)-6 \delta$. Since $\left(\gamma v_{0} \cdot x\right)_{p} \leq \delta$, similarly, $d\left(\gamma v_{0}, x\right) \geq d\left(\gamma v_{0}, p\right)+d(p, x)-2 \delta$.

By the triangle inequality, on the other hand, $d\left(\gamma v_{0}, y\right) \leq d\left(\gamma v_{0}, p\right)+d(p, y)$, and $d\left(\gamma v_{0}, x\right) \leq d\left(\gamma v_{0}, p\right)+d(p, x)$. One can thus estimate $|d(p, x)-d(p, y)|-2 \delta \leq$ $\left|d\left(\gamma v_{0}, x\right)-d\left(\gamma v_{0}, y\right)\right| \leq|d(p, x)-d(p, y)|+6 \delta$.

By assumption $\star_{0}$ on $x$ and $y$, and since $p$ is on $\left[v_{0}, x\right]$ and $\delta$-close to $\left[v_{0}, y\right]$, one has $|d(p, x)-d(p, y)| \leq 2 \delta$. This ensures that $\left(\gamma^{-1} x, \gamma^{-1} y\right)$ satisfy $\star_{10 \delta}$.

By assumption, $d\left(\gamma v_{0}, x\right) \leq R(n)-50 \delta$; hence there exists a path $c:[0, n] \rightarrow X$ from $x$ to $y$, such that for all $t, d\left(\gamma v_{0}, c(t)\right) \geq \min \left\{d\left(\gamma v_{0}, x\right), d\left(\gamma v_{0}, y\right)\right\}-C-15 \delta$; in particular, $d\left(\gamma v_{0}, c(t)\right) \geq d\left(\gamma v_{0}, x\right)-C-25 \delta$. 
We now need to control $d\left(v_{0}, c(t)\right)$ for all $t$. Changing $y$ by $c(t)$ in the argument above provides the estimation $d(p, c(t))-d(p, x) \geq d\left(\gamma v_{0}, c(t)\right)-d\left(\gamma v_{0}, x\right)-6 \delta$.

One also easily gets that $d\left(v_{0}, c(t)\right)-d\left(v_{0}, x\right) \geq d(p, c(t))-d(p, x)-2 \delta$; thus, $d\left(v_{0}, c(t)\right)-d\left(v_{0}, x\right) \geq d\left(\gamma v_{0}, c(t)\right)-d\left(\gamma v_{0}, x\right)-6 \delta \geq-C-35 \delta$, as required.

Corollary 4.5. Let $n \geq K$. Assume that $\ddagger(10 \delta, n)(x, y)$ holds for all pairs of vertices $(x, y)$ in $B_{v_{0}}(R(n))$ satisfying $\star_{10 \delta}$.

Assume furthermore that all parabolic subgroups of $G$ are abelian (so that $X$ satisfies the conclusion of Lemma 2.16).

For all pairs of points $(x, y)$ in $X$ satisfying $\star_{0}$, such that $d\left(x, v_{0}\right) \geq R(n)$, the property $\ddagger(0, n)$ is satisfied.

Proof. By Lemma 4.4 if $x$ and $y$ are in $X_{k}$, then they satisfy $\ddagger(0, n)$. On the other hand, if $x$ is not in $X_{k}$, then it is at depth at least $k$ in a horoball, and since $y$ is at a distance at most $M<k$ from $x$, it is in the same horoball. Lemma 2.16 then guarantees the result.

Corollary 4.6. Let $G$ be a relatively hyperbolic group. Assume that the parabolic subgroups are abelian, and let $X$ be a proper hyperbolic space associated to $G$ as above, and let $K$ be as in Section 2.3 .

If $G$ has connected boundary, and has no proper peripheral splitting, then there exists $n \geq K$, such that $\ddagger(10 \delta, n)$ holds for all pairs $(x, y)$ in $B_{v_{0}}(R(n))$ satisfying $\star 10 \delta$.

If there exists $n \geq K$, such that $\ddagger(10 \delta, n)$ holds for all pairs $(x, y)$ in $B_{v_{0}}(R(n))$ satisfying $\star_{10}$, then $\partial G$ is connected.

In fact, what one really needs is that the parabolic subgroups satisfy the conclusion of Lemma 2.16, in order to apply the previous corollary, and that they are one-ended (or two-ended), finitely presented, and without infinite torsion subgroup, in order to apply Bowditch's results.

Proof. If $G$ has connected boundary, and has no proper peripheral splitting, then by Theorem 3.3 (1-2), $\partial G$ has no global cut point. Therefore, by Lemma 4.2, there exists $n$ (which can be assumed to be at least $K$ ) such that $\ddagger(10 \delta, n)$ holds for all pairs $(x, y)$ in $B_{v_{0}}(R(n))$ satisfying $\star_{10} \delta$.

Conversely, by Corollary 4.5, and Lemma 4.1, $\partial G$ is connected.

\section{Algorithms}

\subsection{Tools.}

Proposition 5.1. There is a procedure that, given a relative presentation of a relatively hyperbolic group $G$ with abelian parabolic subgroups (so that the intersection of the generating set with each parabolic subgroup is sensible), terminates if the boundary $\partial G$ is connected, without global cut point, and only if $\partial G$ is connected.

Proof. First compute the constant $\delta$ for the space $X$, and set $n=K$.

Next, compute the ball $B_{v_{0}}(R(n)+n)$ in $X$. Check whether $\ddagger(10 \delta, n)(x, y)$ holds for all pairs of vertices $(x, y)$ in $B_{v_{0}}(R(n))$ satisfying $\star_{10 \delta}$ (there are finitely many such pairs). If this is so, then by Corollary 4.6, $G$ has connected boundary, so the algorithm is told to stop there. If not, increment $n$ by 1 , and restart this procedure. 
By Corollary 4.6, if the procedure does not stop, either the boundary $\partial G$ is disconnected, or it has a global cut point. On the other hand, if this procedure does stop, then by Corollary 4.6, $\partial X$ is connected.

Proposition 5.2. There is a procedure that, given a finite presentation of a group, with a solution to the word problem, terminates if and only if the group has more than one end.

Proof. Enumerate the presentations of the group by Tietze transformations. Each time one of them exhibits a presentation of an amalgamated free product, or of an HNN extension, start in parallel the computation of the multiplication table of the edge group, using the solution to the word problem. One such computation eventually terminates if and only if the edge group is finite. For each of the terminating computations, check whether the splitting is nontrivial (if the generators of a vertex group are all in the edge group). Since the edge group is finite, and the vertex group is given with a finite set of generators, this involves finitely many applications of the algorithm to solve the word problem.

The whole procedure is told to terminate when it has found a nontrivial splitting over a finite group.

Proposition 5.3. There is a procedure that, given a relative presentation of a relatively hyperbolic group $G$, along with a solution to the word problem for $G$, enumerates the peripheral splittings of $G$.

Proof. Enumerate the presentations of the group by Tietze transformations. Each time one of them exhibits a presentation of a finite bipartite graph of groups, start in parallel the following procedure.

For each vertex of a given color, of generators $c_{1}, \ldots c_{k}$, enumerate the conjugates $c_{1}^{g}, \ldots, c_{k}^{g}$, for $g \in G$, and check, with a solution to the word problem, whether one of these families coincides with the generators of a given parabolic subgroup. If there is a bijective such correspondence between vertices of one color and given representatives (up to conjugacy) of parabolic subgroups, then the presentation exhibits a peripheral splitting. It is then output in the list.

Given any peripheral splitting, a presentation exhibiting it will appear eventually in the enumeration, and thus will be eventually spotted by the algorithm.

\subsection{Results and conclusion.}

Theorem 5.4. There exists an algorithm whose input is a finite presentation of a relatively hyperbolic group with abelian parabolic subgroups and that outputs the answer to the question of the connectivity of its boundary.

Proof. According to Proposition [3.1, $\partial G$ is disconnected if and only if $G$ splits nontrivially over a finite group relative to $\mathbf{P}$.

First of all, we run the procedure from Proposition 5.2. If there is a nontrivial splitting over a finite subgroup, we will find it. We can run a procedure on each such splitting which terminates if each element of $\mathbf{P}$ is elliptic (i.e. conjugate into a vertex group). Thus, if the boundary $\partial G$ is disconnected, we will eventually discover this. We now need a second procedure to run in parallel which will terminate if the boundary is connected.

Let us run the procedure from Proposition 5.3 (recall that by Theorems 2.8 and 2.10, one can compute the relatively hyperbolic structure of the group, a hyperbolicity constant for $X$, and arbitrarily large balls of $X$, and thus one can run 5.3 . The 
output list of this procedure is a list of graphs of groups (possibly nonterminating). Start this list with the trivial graph of groups with one vertex corresponding to $G$ and no edges.

In parallel, every time a new item appears on the list, let us find (using the algorithm of Theorem 2.8) a relative presentation for the vertex groups, where any adjacent edge groups are parabolic, using the algorithm of Theorem 2.8. Then, let us check whether the splitting contains a nontrivial splitting over a finite group (the edge groups are given in terms of the presentations of the parabolic subgroups, which are finitely generated abelian; thus it is easy to check whether they are finite). If yes, announce that the group has disconnected boundary (this is true by Proposition 3.1). If no, then let us run the procedure from Proposition 5.1 (checking connectedness of the boundary, modulo the presence of cut points) on each of the vertex groups. If the algorithm from Proposition 5.1 has stopped on every nonparabolic vertex group of a given splitting, then announce that the boundary is connected.

We claim that if the procedure from Proposition 5.1 terminates on each nonparabolic vertex group of a given splitting, then the boundary is indeed connected (as our algorithm announces). For, in this case all edge groups are infinite, and the boundaries of the vertex groups are connected (because the algorithm from Proposition 5.1 is assumed to have stopped). One can easily see in this case that $\partial G$ is connected, since it is obtained by gluing together boundaries of vertex groups along points fixed by infinite parabolic edge groups, and then compactifying by the boundary of the Bass-Serre tree (see [7, for instance).

It remains to check that the algorithm presented always terminates. If the boundary is disconnected, then by Proposition 3.1] there is a peripheral splitting over a finite group, and it will be listed in the first procedure. On the other hand, if $\partial G$ is connected, by the accessibility Theorem 3.4, the procedure from Proposition 5.3 eventually finds a peripheral splitting in which the nonparabolic vertex groups have connected boundaries without global cut points. Therefore, by Corollary 4.6, on this particular entry at least, the algorithm from Proposition 5.1 terminates.

Proof of Theorem 1.2, By Theorem 2.8, one can find a collection of abelian subgroups $\mathbf{P}=\left\{P_{1}, \ldots, P_{n}\right\}$ (with presentations) of $G$, relative to which it is hyperbolic. One can identify the groups $P_{i}, i \in I$ that are virtually cyclic, and it is classical that $G$ is still relatively hyperbolic with respect to $\mathbf{P}^{\prime}=\mathbf{P} \backslash\left\{P_{i}, i \in I\right\}$, which consists this time of one-ended abelian subgroups. Now, by Proposition 3.1, $G$ admits a splitting over a finite group if and only if its boundary (for this latter structure $\left.\left(G, \mathbf{P}^{\prime}\right)\right)$ is connected. Theorem 5.4 can then be applied to $\left(G, \mathbf{P}^{\prime}\right)$, giving the result.

Proof of Theorem 1.3. Let us run the procedure of Theorem 1.2, if the group has one end, there is nothing to add; if it has several ends, the output of the algorithm is a splitting of the group as a graph of groups, with only finite edge groups. The Dunwoody decomposition is a refinement of this splitting; thus we need to run the same procedure on each vertex group. This process eventually stops, as accessibility is ensured.

Proof of Theorem 1.4. It suffices to identify the trivial edge groups in the Dunwoody decomposition, that is, the output of Theorem 1.3. Every edge group in this 
decomposition is described in the output of Theorem 1.3 as a subgroup of a parabolic group of $G$ for which we know a presentation, and that is finitely generated abelian. Thus it is easy to decide whether it is trivial.

\section{REFERENCES}

[1] M. Bestvina and G. Mess, "The boundary of negatively curved groups", J. Amer. Math. Soc. 4 (1991), 469-481. MR 1096169 (93j:20076)

[2] B. Bowditch, "Connectedness properties of limit sets", Trans. Amer. Math. Soc. 351 (1999), no. 9, 3673-3686. MR1624089 (2000d:20056)

[3] B. Bowditch, "Boundaries of geometrically finite groups", Math. Z. 230 (1999), no. 3, 509527. MR 1680044 (2000b:20049)

[4] B. Bowditch, "Peripheral splittings of groups", Trans. Amer. Math. Soc. 353 (2001), 40574082. MR1837220 (2002e:20080)

[5] B. Bowditch, "Relatively hyperbolic groups", preprint.

[6] M.R. Bridson and A. Haefliger, Metric spaces of non-positive curvature, Springer-Verlag, Berlin, 1999. MR,1744486 (2000k:53038)

[7] F. Dahmani, "Combination of convergence groups" Geom. \& Topol. 7 (2003), 933-963. MR2026551 (2005g:20063)

[8] F. Dahmani, "Finding relative hyperbolic structure", preprint.

[9] F. Dahmani and D. Groves, "The isomorphism problem for toral relatively hyperbolic groups", preprint. Available at http://arxiv.org/abs/math.GR/0512605.

[10] M. Dunwoody, The accessibility of finitely presented groups, Invent. Math. 81 (1985), 449457. MR807066 (87d:20037)

[11] C. Druţu and M. Sapir, Tree-graded spaces and asymptotic cones of groups, Topology 44 (2005), 959-1058. MR2153979 (2006d:20078)

[12] B. Farb, Relatively hyperbolic groups, Geom. Funct. Anal. 8 (1998), 810-840. MR1650094 (99j:20043)

[13] V. Gerasimov, Detecting correctedness of the boundary of a hyperbolic group, unpublished.

[14] M. Gromov, Hyperbolic groups, in Essays in group theory (S.M. Gersten, ed.), Springer Verlag, MSRI Publ. 8 (1987), 75-263. MR919829 (89e:20070)

[15] D. Groves and J.F. Manning, Dehn filling in relatively hyperbolic groups, preprint. Available at arxiv.org/math.GR/0601311.

[16] O. Kharlampovich, and A. Miasnikov, Effective JSJ decompositions, in Groups, languages, algorithms, Contemp. Math 378 (2005), 87-212. MR2159316 (2006m:20045)

[17] D. Rebbechi "Algorithmic properties of relatively hyperbolic groups". Ph.D. thesis (2001).

Laboratoire E. Picard, Université Paul Sabatier, F-31062 Toulouse, France

E-mail address: dahmani@picard.ups-tlse.fr

Department of Mathematics, California institute of Technology, Pasadena, CaliFORNIA 91125

E-mail address: groves@caltech.edu

Current address: Department of Mathematics and Computer Science, University of Illinois at Chicago, Chicago, Illinois 60607-7045

E-mail address: groves@math.uic.edu 